\title{
Duración, intensidad y contexto de clases de Educación Física con el modelo educativo por competencias en Educación Primaria
}

Javier Arturo Hall-López y Paulina Yésica Ochoa-Martínez

Facultad de Deportes, Universidad Autónoma de Baja California (UABC), México

Email autor correspondencia: pochoa@uabc.edu.mx

RESUMEN: El objetivo de la investigación fue evaluar el tiempo, intensidad y contexto de treinta dos sesiones de educación física realizadas bajo el modelo educativo por competencias de la Secretaria de Educación Pública, en escuelas primarias de la ciudad de Mexicali, Baja California (México). El estudio fue transversal, descriptivo y como metodología de evaluación se realizó mediante observación por el sistema para observar el tiempo de instrucción de actividad física (SOFIT). El resultado del tiempo promedio de la sesión fue de $41.8 \pm 9.3$ minutos, la distribución porcentual de actividad física moderada a vigorosa resulto en $40.5 \%$ y el predominio de contexto fue la administración, concluyendo que no hay promoción de actividad física moderada a vigorosa. La evaluación realizada aporta elementos constructivos para retroalimentar la manera de impartir la clase con estrategias didácticas involucrando al alumno en acciones motrices de intensidad moderada a vigorosa por lo menos el $50 \%$ de la clase.

PALABRAS CLAVE: Educación Física, SOFIT, Educación Primaria.

\section{Duration, intensity and context of Physical Education classes with the educational model by competences in Primary Education}

\begin{abstract}
The objective of the research was to evaluate the time, intensity and context of thirty two physical education sessions conducted under the educational model by competencies of the Ministry of Public Education, in primary schools in the city of Mexicali, Baja California (Mexico). The study was cross-sectional, descriptive and as an evaluation methodology was carried out by observation by the system to observe the instructional time of physical activity (SOFIT). The results of the average session time were $41.8 \pm 9.3$ minutes, the percentage distribution of moderate to vigorous physical activity resulted in $40.5 \%$ and the predominance of context was the administration, concluding that there is no promotion of moderate to vigorous physical activity. The evaluation carried out provides constructive elements to provide feedback on how to teach the class with didactic strategies involving the student in motor actions of moderate to vigorous intensity at least $50 \%$ of the class.
\end{abstract}

KEY WORDS: Physical Education, SOFIT, Primary Education. 
Este artículo está publicado como capítulo en el libro: Hall, J. A., Ochoa, P. Y., y SáenzLópez, P. (2018). Intensidad, salud, motivación y adherencia en Educación Física. Huelva: Servicio de publicaciones de la Universidad de Huelva.

\section{INTRODUCCIÓN}

Actualmente en México el programa de educación física con el modelo educativo por competencias (SEP, 2008) implementa en los estudiantes un carácter abierto de desarrollo motor en tres grupos, manifestación global de la corporeidad, expresión y desarrollo de habilidades y destrezas motrices y control de la motricidad para el desarrollo de la acción creativa (SEP, 2008), mediante el movimiento con el propósito central de que el alumno incorpore capacidades que incluyen conocimientos, actitudes, habilidades y destrezas. Además, se pretende que sea capaz de controlar su cuerpo a fin de producir respuestas motrices adecuadas ante las distintas situaciones que se le presenten, tanto en la vida escolarizada como en los diversos contextos donde convive y edifique desempeños motrices complejos (Lleixá, 2007, SEP, 2008). El profesor evalúa este proceso de diversas maneras, de acuerdo al bloque del programa, con un instrumento basado en escala cualitativa (excelente, muy bien, bien, regular), donde se observa calidad de movimientos se registra la calidad creativa como diversidad, originalidad e ideas expuestas en movimiento del producto creativo de expresión corporal, la observación del desempeño motriz en los patrones básicos de movimiento y verificación de formas de organización en los juegos cooperativos, la observación del número de modificaciones a los juegos propuestos, la observación de la comunicación en equipo, diversidad y calidad de ideas, sincronización de movimiento, las propuestas de coevaluación en relación al producto final (variedad de movimientos, desplazamientos, distribuciones asimétrica, simétrica), las propuestas de autoevaluación de acuerdo al desempeño en la solución de problemas, observación de tolerancia, respeto al momentos de interactuar, el registro por parte de los alumnos propuestas originales que más les agradó, las sugerencias de una autoevaluación de lo aprendido y en toda la educación primaria con respecto a la educación física, el registro del nivel de competencia motriz de los alumnos y la solicitud de un collage de cada alumno puedan explicarse ellos mismo y puedan decir: este soy yo (López-Pastor et al., 2006, SEP, 2008).

Se tiene antecedentes de investigaciones realizadas en México (JenningsAburto et al., 2009; Pérez-Bonilla, 2009), donde se evaluó la duración, intensidad y el contexto de la clase de educación física en primaria, dichos estudios evaluaron clases de educación física (EF) bajo el enfoque motriz de integración dinámica, el cual privilegia las capacidades físicas condicionales y coordinativas del alumno y por consiguiente la eficacia del movimiento (fundamentos técnicos deportivos). Los resultados mostraron una duración de la clase por debajo de los 50 minutos oficiales, una intensidad moderada a vigorosa por debajo del $50 \%$ de acuerdo a los estándares internacionales como la United States National Association for Sport and Physical Education (NASPE) y un contexto en el cual el administrar la clase y el desarrollo de habilidades motrices conlleva gran parte de la totalidad de la clase, concluyendo en que la clase de EF no se promueve la actividad física moderada a vigorosa y se requiere revisar la manera de impartirla (NASPE, 2004).

Por lo anterior nuestro propósito de investigación es identificar la duración, intensidad y contexto de la clase de educación física con el modelo educativo por competencias de cuarto, quinto y sexto grado en escuelas primarias de Mexicali (Baja California, México) y compararla con referencias de estudios mexicanos donde 
evaluaron duración, intensidad y contexto de clases de educación física por el modelo motriz de integración dinámica.

\section{MÉTODO}

El diseño de la investigación fue de corte transversal descriptivo, con muestra no probabilística por conveniencia.

\subsection{Participantes}

Para evaluar a 32 profesores de educación física (EF) se seleccionaron clases de educación física de cuarto, quinto y sexto; eligiendo para el tiempo de instrucción a 4 estudiantes de cada clase para observarlos en secuencia rotatoria, durante 12 intervalos de 20 segundos cada uno, repitiéndose las observaciones durante toda la clase, siguiendo el audio del System for Observing fitness and Instruction Time (SOFIT; McKenzie, Sallis, y Nader, 1991) en un reproductor MP3 Samsung YP-U6AB. En concreto, las clases de EF de los 32 docentes evaluados pertenecían a escuelas primarias del subsistema federal de la ciudad de Mexicali (Baja California, México).

\subsection{Instrumento}

El instrumento que se utilizó para evaluar el tiempo, intensidad y contexto de la clase de educación física fue el SOFIT (System for Observing fitness and Instruction Time; McKenzie et al., 1991) sistema para observar el tiempo de instrucción de actividad física, por sus siglas en inglés. Para evaluar a los 32 profesores de educación física se seleccionaron clases de educación física de cuarto, quinto y sexto eligiendo para el tiempo de instrucción a 4 estudiantes de cada clase para observarlos en secuencia rotatoria, durante 12 intervalos de 20 segundos cada uno, repitiéndose las observaciones durante toda la clase, siguiendo el audio de SOFIT (McKenzie et al., 1991) en un reproductor MP3 Samsung YP-U6AB. En la evaluación SOFIT se usaron códigos para clasificar los niveles de actividad, los cuales permitieron estimar la energía gastada asociada con la actividad física. Este procedimiento se ha usado para evaluar los efectos del entrenamiento relacionado con el currículum de educación física. Los códigos se clasifican en cuatro: 1) acostado, 2) sentado, 3) parado, 4) caminando, y 5) muy activo que corresponde a correr o a cuando el estudiante realiza más actividad física que la que corresponde al caminar ordinario. Estos códigos han sido calibrados monitoreando los latidos del corazón (McKenzie et al., 1991), y el sistema ha sido validado usando acelerómetros Caltrac. (McKenzie et al., 1994).

Un segundo aspecto que evalúa el SOFIT simultáneamente con la actividad física del estudiante es el contexto de la clase. Este contexto se codifica en siete categorías, $\mathrm{M}=$ Administración, $\mathrm{P}=$ Conocimiento específico, $\mathrm{K}=$ Conocimiento general, $\mathrm{F}=$ Acondicionamiento físico, $\mathrm{S}=$ Desarrollo de habilidades, $\mathrm{G}=$ Juego, $\mathrm{O}=$ Otros.

El tiempo de la clase de educación física se cronometro Casio HS3W estableciendo el inicio y fin de la clase de educación física de acuerdo al horario establecido para la sesión de 50 minutos.

\subsection{Procedimiento.}

La presente investigación fue llevada a cabo entre marzo y abril de un curso escolar. Los datos fueron recogidos de 32 sesiones de educación física en escuelas 
primarias del subsistema federal de la ciudad de Mexicali (Baja California, México). Como fase previa, para poder llevar a cabo el presente estudio, fue necesario solicitar la autorización de los directivos y los profesores de educación física de cada institución, explicando de manera verbal y por escrito los propósitos y beneficios de la realización del presente estudio, así como el impacto del mismo. Los profesores participantes fueron seleccionados a partir de una invitación personal autorizando participar voluntariamente.

\subsection{Análisis de los datos}

Para el análisis de los datos se utilizó el programa SPSS, donde se realizaron cálculos para su procesamiento estadístico obteniendo de manera descriptiva media, desviación estándar, con los resultados obtenidos se realizaron gráficas en el software Excel, que reflejan los datos más significativos de esta investigación.

\section{RESULTADOS}

El tiempo promedio utilizado por los profesores para las sesiones de educación física fue de $41.8 \pm 9.3$ minutos, lo que está por debajo de lo establecido por la Secretaria de Educación Pública. En la Figura 1 se muestra la distribución porcentual de la intensidad de actividad física estableciendo un índice de actividad física moderada a vigorosa, donde en promedio fue de $40.5 \%$, la cual no cumple con los estándares establecidos por el United States' National Association for Sport and Physical Education (NASPE).

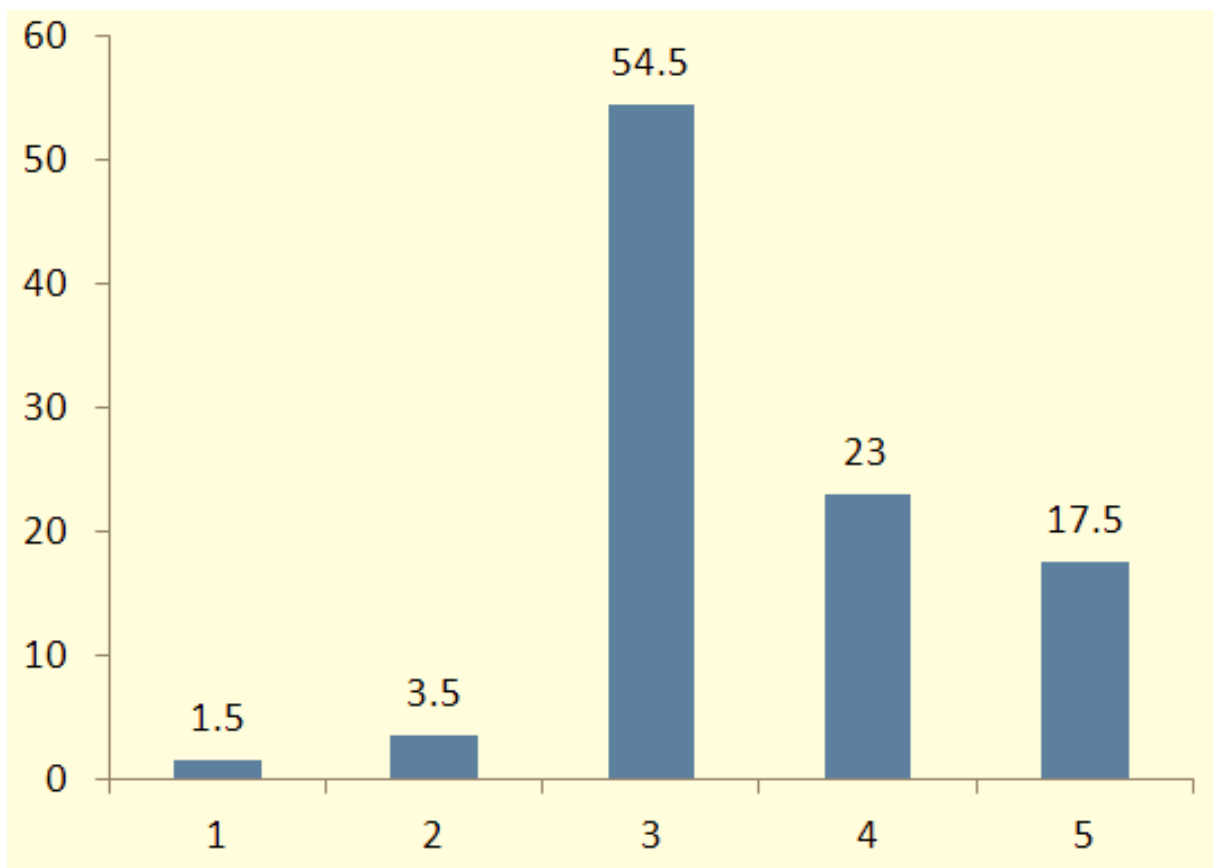

Figura 1. Distribución porcentual de la intensidad de actividad física de 32 sesiones de EF en escuelas primarias de la ciudad de Mexicali (Baja California).

La Figura 2 muestra el contexto de la clase, derivado de la planeación del profesor donde destaca en mayor medida al apartado M (Contenido general) donde el profesor 
utilizó su tiempo de clase en contenidos general incluye la transición, administración y descanso.

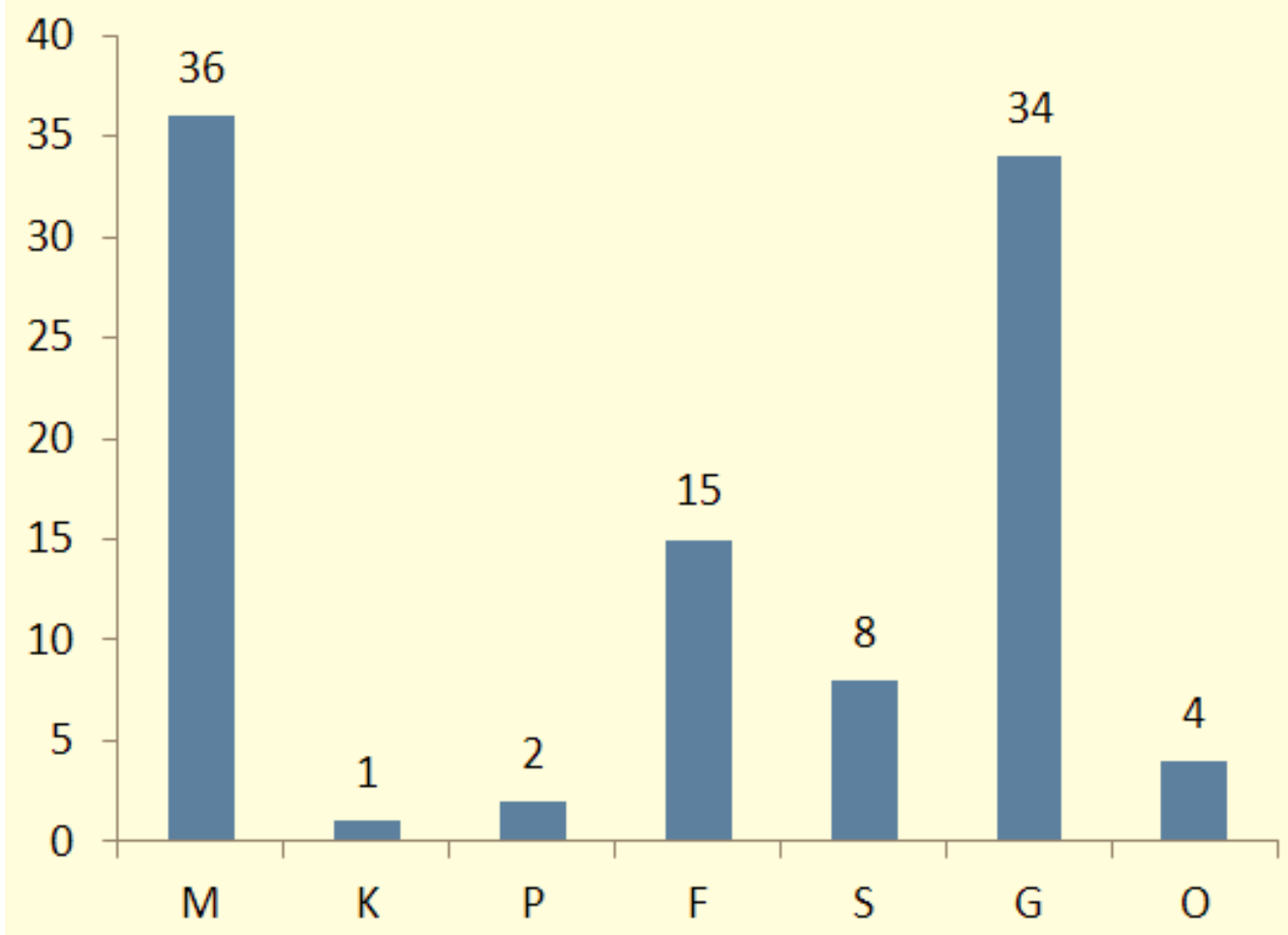

Figura 2. Distribución porcentual del contexto de 32 sesiones de EF en escuelas primarias de la ciudad de Mexicali (Baja California). Nota: $\mathrm{M}=$ Administración, $\mathrm{P}=$ Conocimiento específico, $\mathrm{K}$

$=$ Conocimiento general, $\mathrm{F}=$ Acondicionamiento físico, $\mathrm{S}=$ Desarrollo de habilidades, $\mathrm{G}=$ Juego, $\mathrm{O}=$ Otros

\section{DISCUSIÓN}

Los datos obtenidos de esta investigación indicaron un promedio de tiempo promedio utilizado por los profesores para las sesiones de educación física fue de 41.8 \pm 9.3 minutos, lo cual refiere mayor tiempo a evaluaciones de clases de educación física realizadas que otros estudios con el programa motriz de integración dinámica refieren 37.3 minutos (Pérez-Bonilla, 2009) y 39.8 minutos (Jennings-Aburto et al 2009), lo que está por debajo de los 50 minutos establecidos para las clases de educación física por la Secretaria de Educación Pública de México. En esta línea, las sesiones evaluadas en la presente investigación indican una pérdida de tiempo en actividades que no son propiamente de la clase de ecuación física, como la preparación del material, el traslado de los alumnos al lugar de la clase, que el profesor no empieza o termina la clase en el tiempo establecido, etc.

La intensidad de actividad física moderada a vigorosa en las 32 sesiones de las clases de EF evaluadas al cuantificar en el mismo rubro los niveles 4 y 5 representan el $40.5 \%$ del tiempo de la clase, presentándose inadecuado de acuerdo a los estándares establecidos por el United States' National Association for Sport and Physical Education (NASPE). Hay que resaltar que en el presente estudio se presentó 
que del promedio del tiempo de las clases de educación física evaluadas, el $54.5 \%$ de la clase estuvieron parados reflejando actividades pasivas al utilizar estrategias en las cuales las formaciones predominaban y las oportunidades de participar eran menos probables limitando la esencia del movimiento a la clase de educación física (Santa María, Laíño, y Pintamalli, 2011). Estudios con igual diseño metodológico en clases de educación física con el programa motriz de integración indican porcentajes de actividad física moderada a vigorosa de 38.2 \% (Pérez-Bonilla, 2009) y 29.2 \% (Jennings-Aburto et al., 2009).

En cuanto al contexto de la clase de educación física, en las estrategias didácticas implementadas por el profesor, se puede observar como el profesor tomó significativamente la mayor cantidad del tiempo empleado en el contenido general (codificado con M) para administrar la clase de educación física (con un 36 \%), refiriendo al tiempo empleado en administrar y organizar las actividades relacionadas con instrucciones tales como la formación de equipos, cambio de equipos o cambio de actividades dentro de la clase.

Con los resultados obtenidos, en los sujetos evaluados, se puede afirmar que, a través del sistema para observar el tiempo de instrucción de actividad física (SOFIT), puede analizarse de manera cuantitativa la duración, intensidad y contexto de la clase impartida por el profesor de educación física. De esta forma, se puede obtener información para retroalimentar de manera constructiva la intervención pedagógica del docente de educación física; dando como recomendación buscar estrategias que coadyuven a maximizar el uso del tiempo de la clase de educación física e implementar estrategias didácticas mantengan a los estudiantes en intensidades moderadas a vigorosas por lo menos el $50 \%$ de la clase, utilizando contextos de administración de la clase en los cuales se involucre el movimiento del alumno. En este sentido, las variables evaluadas de tiempo, intensidad y contexto de la clase de educación física, salieron por debajo de los estándares (NASPE, 2004), por lo que son necesarias intervenciones educativas para mejorar la efectividad de los docentes de EF y que consigan impartir clases a unas intensidades de AF más adecuadas para la salud de su alumnado.

\section{REFERENCIAS}

Lleixá, T. (2007). Educación física y competencias básicas. Contribución del área a la adquisición de las competencias básicas del currículo. Tándem: Didáctica de la Educación Física, 23, 31-37.

López-Pastor, V., Monjas, R., Gómez, J., López-Pastor, E., Martín, J., González, J, Barba, J., Aguilar, R., González, M., Heras, C., Martín, M., Manrique, J., Subtil, P., y Marugán, L. (2006). La evaluación en educación física. Revisión de los modelos tradicionales y planteamiento de una alternativa: la evaluación formativa y compartida. Retos Nuevas Tendencias en Educación Física Deporte y Recreación, 10, 31-41.

Jennings-Aburto, N., Nava, F., Bonvecchio, A., Safdie, M., González-Casanova, I., Gust, T., y Rivera, J. (2009). Physical activity during the school day in public primary schools in Mexico City. Salud Pública Mex, 51, 141-147.

National Association for Sport and Physical Education (2004). Physical activity for children: a statement of guidelines (2nd ed). Reston, VA: National Association for Sport and Physical Education. 
McKenzie, T., Sallis, J., y Nader, P. (1991). SOFIT. System for Observing Fitness Instruction Time. Journal of Teaching in Physical Education, 11, 195-205.

McKenzie, T. L., Strikmiller, P. K., Stone, E. J., Woods, S. E., Ehlinger, S. S., Romero, K. A., y Budman, S. B. (1994). CATCH: Physical activity process evaluation in a multicenter trial. Health Education Quarterly, Supl. 2, 73-S89. doi: $10.1177 / 10901981940210$ s106

Pérez-Bonilla, A. M. (2009) Impacto de la clase de educación física sobre la actividad moderada y vigorosa en niños de primaria. Revista Mexicana de Investigación en Cultura Física y Deporte, 1(1),150-172.

Santa-María, C. J., Laíño F. A, y Pintamalli, J. C. (2011). Gasto energético en las clases de educación física de escuelas primarias y secundarias de la ciudad de Buenos Aires. ReCAD - Revista electrónica de Ciencias Aplicadas al Deporte, 4(12), 1-13.

Secretaria de Educación Pública (2008). Programas de Estudio 2009 y Guías de Actividades Sexto Grado.

Secretaria de Educación Pública (2008). El Plan en Etapa de Prueba para Educación Física en Primarias. 